\title{
Onychomycosis Caused by Malassezia pachydermatis in a Dog
}

\author{
Andréia Spanamberg', David Driemeier², Luciana Sonne² \& Laerte Ferreiro
}

\begin{abstract}
Background: Malassezia species are commensal skin organisms of warm-blooded vertebrates that can act as opportunistic pathogens. Malassezia pachydermatis is of importance in both veterinary and human medicine. Recognised initially as causes of infection of the skin, they are now known to be superficial commensals as well as potential causes of infections in domestic animals and more serious human conditions such as fungemia. They have also been implicated in the pathogenesis of allergic and other inflammatory diseases. Onychomycosis is defined as fungal infection of the claw (nail disease) caused by dermatophytes (Microsporum and Trichophyton genus), non-dermatophyte molds (hyaline and dematiaceous) and/or yeasts. The objective of this work was to report a case of canine onychomycosis by Malassezia pachydermatis based on reference standard diagnostics of this pathology.

Case: A 9-year-old male West Highland White Terrier presented history of claw abnormality: brown staining and partly brittle. The dog has no apparent history of trauma on the affected claw and no skin disease was reported. Direct examination of claw was performed using the tape strip technique revealed Malassezia organisms. Nail fragments were cultured onto Sabouraud Dextrose Agar with Chloramphenicol and Cycloheximide. After 10 days of incubation $\left(32^{\circ} \mathrm{C}\right)$ nail fragments allowed the isolation of pure colonies of Malassezia pachydermatis. The histopathological evaluation was performed by Sector of Veterinary Clinical Pathology, Faculdade de Veterinária (FAVET/UFRGS) and fungal infection due to Malassezia pachydermatis was confirmed by histopathologic examination (Hematoxylin and eosin and Grocott's methenamine silver stain) of the nail, that revealed abundant yeasts (blastoconidia).

Discussion: Fungal infection of the skin, hair and nails are common and are primary caused by the dermatophyte molds. Non-dermatophyte molds isolated from nails constitute a long list, but only a few species cause onychomycoses. These include Scopulariopsis brevicaulis, Fusarium sp., Acremonium spp., Aspergillus spp., Penicillium spp. Yeasts like Malassezia and Candida spp. usually are saprobics and usually represent incidental contaminants associated with a non-sterile specimen at mycological culture, however in some cases they may parasite the nails and become an etiological agent of the disease. M. pachydermatis, normally present on the skin and in the ear canal of dogs and cats, can act as opportunistic pathogenic microbe and frequently causes dermatitis and otitis in mammals. In the case of onychomycosis, there is little evidence that Malassezia yeasts are implicated in nail plate invasion, although this may be a rare occurrence. All species of the genus are obligatory lipid-dependent forms, with the exception of M. pachydermatis, as well as absence of keratinolytic ability. Malassezia organisms were commonly retrieved from the subungual claw fold region of normal healthy dogs (should be considered resident microflora of the normal canine claw fold). Some authors suggests that high numbers of yeast can be detected on cytological evaluation of the skin surface of the canine claw fold from allergic dogs in both the absence and presence of concurrent signs of pruritus and paronychia.
\end{abstract}

Keywords: dog, nail, Malassezia pachydermatis, onychomycosis. 


\section{INTRODUCTION}

Diseased claws will predispose to trauma, abnormal locomotion, pain, lameness, and pododermatitis. Various medical terms are used to describe the claw lesion, being onychomycosis the term used when a fungal infection occurs. Dogs with Malassezia infections show brown-red discoloration of the claw with brown-colored waxy exudate on the proximal aspects of the claws [1,3].

Several species are currently recognized in the genus Malassezia, most of which are lipid-dependent (M. furfur, M. globosa, M. obtusa, M. restricta, M. sloofiae, M. sympodialis, M. dermatis, M. nana, $M$. japonica, M. yamatoensis, $M$. equina, M. caprae, $M$. cunniculi) and only one non lipid-dependent (M. pachydermatis). Genomic analysis has allowed a more reliable classification of the yeasts that constitute the genus Malassezia [2,7].

This yeast has been isolated from most domestic animals, wild animals and free living in various regions of the world. The finding of an apparent specificity by some hosts suggests a possible increase in the discovery of new species of Malassezia in other animal species [1,7].

Cytology, culture and histopathology have already been used for the quantitative and qualitative research of $M$. pachydermatis obtained from various regions of the body, both healthy animals and those with dermatopathies, but it was not possible to standardize or determine the most effective. Mycological culture continues to be the reference standard for evaluating and comparing results, especially regarding the sensitivity and specificity of alternative techniques [7]. The aim of this study was to report a case of canine onychomycosis by Malassezia pachydermatis based on the standard reference diagnosis.

\section{CASE}

A 9-year-old male West Highland White Terrier, was referred to a private veterinary clinic, with a history of claw abnormality: brown staining and partly brittle. The dog has no apparent history of trauma on the affected claw and no skin disease was reported. Direct examination of claw was performed using the tape strip technique revealed Malassezia organisms. Nail fragments were cultured onto Sabouraud Dextrose Agar with Chloramphenicol and Cycloheximide ${ }^{1}$. After 10 days of incubation $\left(32^{\circ} \mathrm{C}\right)$ the mycological culture of nail fragments allowed the isolation of pure colonies of Malassezia pachydermatis (Figure 1). The histopathological evaluation was performed by Sector of Veterinary Clinical Pathology, Faculdade de Veterinária (FAVET/UFRGS) and fungal infection due to Malassezia pachydermatis was confirmed by histopathologic examination (Hematoxylin and eosin and Grocott's methenamine silver stain) of the nail (Figure 2), that revealed abundant yeasts (blastoconidia).

\section{DISCUSSION}

Yeasts of the genus Malassezia are commensal skin organisms and can act as opportunistic pathogenic microbe on human and mammalian skin [1], being frequently reported in the Brazilian veterinary literature [9]. Many factors may be associated with the proliferation of Malassezia yeasts in animals, including breed, gender, and age. Nett et al. [11] reported that basset hounds, cocker spaniels, and West Highland white terriers are breeds predisposed to develop Malassezia dermatitis. Alteration of microenvironmental skin conditions connected to a chemical mechanism $(\mathrm{pH}$ modifications), long-term treatments (antibiotics and glucocorticoids), or presence of other bacteria or fungi and coexisting diseases may contribute to Malassezia proliferation $[3,14]$.

Fungal infection of the skin, hair and nails are common and are primary caused by the dermatophyte molds. Non-dermatophyte molds isolated from nails constitute a long list, but only a few species cause onychomycoses. These include Scopulariopsis brevicaulis, Fusarium sp., Acremonium spp., Aspergillus

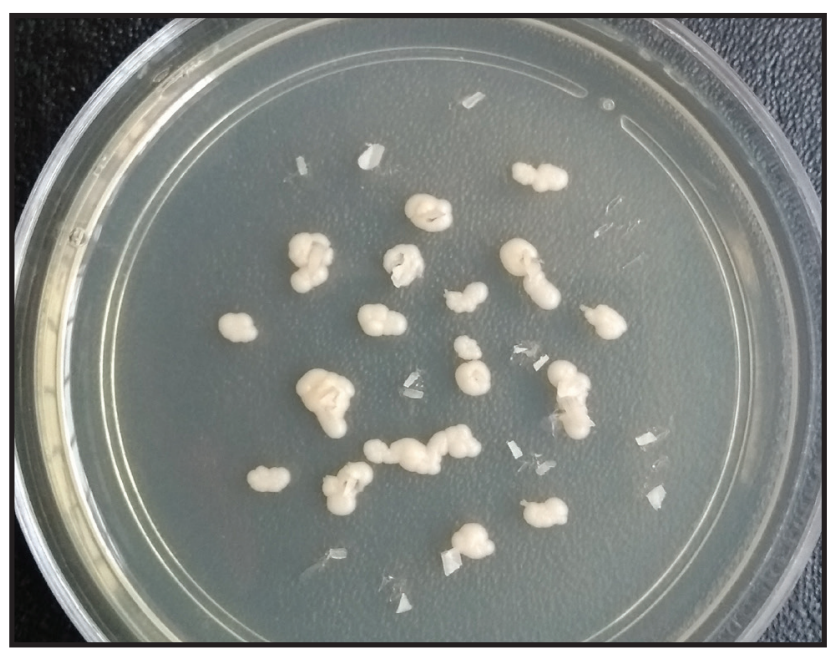

Figure 1. Pure colonies of Malassezia pachydermatis isolated on BD Sabouraud Agar with Chloramphenicol and Cycloheximide. Nail fragments were obtained from a case of onychomycosis in a dog. 


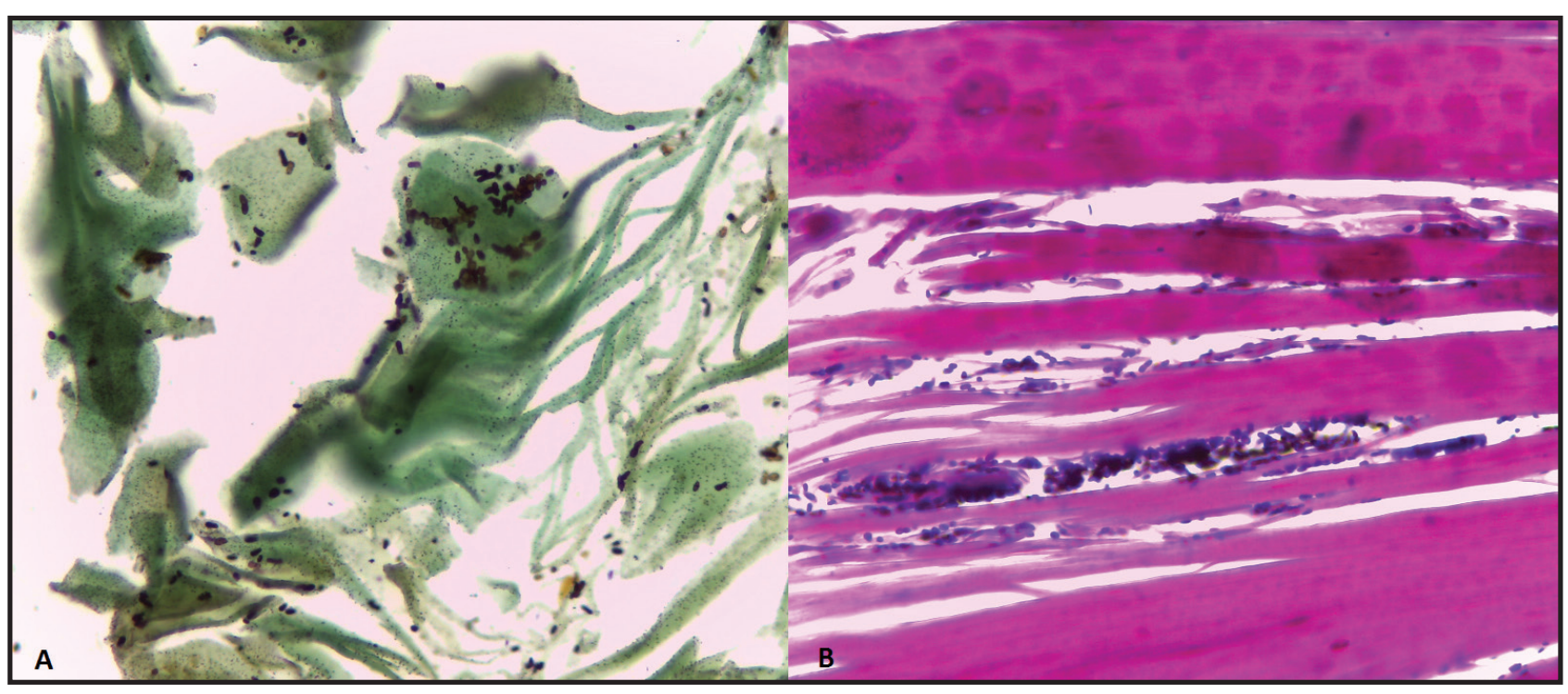

Figure 2. Histopathology of dog's nails with onychomycosis. A- Nail with numerous typical micromorphologic structures (blastoconidia) of Malassezia pachydermatis (Hematoxylin and Eosin, obj.40x). B- Numerous stained blastoconodia (Grocott's methenamine silver stain, obj.40x).

spp., Penicillium spp. Yeasts like Malassezia and Candida spp. are saprophytes and usually represent incidental contaminants associated with a non-sterile specimen at mycological culture, however in some cases they may parasite the nails and become an etiological agent of the disease $[4,7,10]$.

M. pachydermatis, normally present on the skin and in the ear canal of dogs and cats, can act as opportunistic pathogenic microbe and frequently causes dermatitis and otitis in mammals. Physical and chemical factors that enable the attachment of Malassezia and the consequent infection, other conditions that may contribute to Malassezia overgrowth are: hormonal imbalance, keratinization defects (idiopathic seborrhea), excessive production of sebum, bacterial infections, and hypersensitivity processes $[1,8,9]$. In the case of onychomycosis, there is little evidence that Malassezia yeasts are implicated in nail plate invasion, although this may be a rare occurrence [5,6]. All species of the genus are obligatory lipid-dependent forms, with the exception of $M$. pachydermatis, and lack enzymes for keratolytic activity. Malassezia is lipophilic and since nails are not good sources of lipid and since there are no lipids under the nail plate, they do not colonize nails. It is not clear whether Malassezia is a pathogenic agent but therapeutic success has been achieved with antifungals and hence it is not considered as primary pathogen in onychomycosis $[1,12,13]$.

Malassezia organisms were commonly retrieved from the subungual claw fold region of normal healthy dogs (should be considered resident microflora of the normal canine claw fold). Some authors suggests that high numbers of yeast can be detected on cytological evaluation of the skin surface of the canine claw fold from allergic dogs in both the absence and presence of concurrent signs of pruritus and paronychia $[1,8,11]$.

In the case reported here, onychomycosis due Malassezia pachydermatis in a dog was confirmed based mycological and histopathological findings.

\section{MANUFACTURER}

${ }^{1} \mathrm{BD}$ - Becton Dickinson. São Paulo, SP, Brazil.

Declaration of interest. The authors report no conflicts of interest. The authors alone are responsible for the content and writing of the paper.

\section{REFERENCES}

1 Boekhout T., Guého-Kellermann E., Mayser P. \& Velegraki A. 2010. Malassezia and the Skin: Science and Clinical Practice. Berlin: Springer, 319p.

2 Cabañes F.J., Vega S. \& Castellá G. 2011. Malassezia cuniculi sp. nov., a novel yeast species isolated from rabbit skin. Medical Mycology. 49: 40-48. 
3 Cafarchia C., Gallo S., Romito D., Capelli G., Chermette R., Guillot J. \& Otranto D. 2005. Frequency, body distribution, and population size of Malassezia species in healthy dogs and in dogs with localized cutaneous lesions. The Journal of Veterinary Diagnostic Investigation. 17: 316-322.

4 Chowdhary A., Randhawa H.S., Sharma S., Brandt M.E. \& Kumar S. 2005. Malassezia furfur in a case of onychomycosis: colonizer or etiologic agent? Medical Mycology. 43: 87-90.

5 Crozier W.J. \& Wise K.A. 1993. Onicomicosis due to Pityrosporum. Australasian Journal of Dermatology. 34: 109-112.

6 Escobar M.L., Carmona-Fonseca J. \& Santamaria L. 1999. Onicomicosis por Malassezia. Revista Iberoamericana de Micología. 16: 225-229.

7 Ferreiro L., Spanamberg A., Alves S.H. \& Santurio J.M. 2016. Diagnóstico Micológico. In: Larsson C.E. \& Lucas R. (Eds). Tratado de Medicina Externa: Dermatologia Veterinária. São Caetano do Sul: Interbook, pp.18-74.

8 Lo K.L. \& Rosenkrantz W.S. 2016. Evaluation of cytology collection techniques and prevalence of Malassezia yeast and bacteria in claw folds of normal and allergic dogs. Veterinary Dermatology. 27: 279-e67.

9 Machado M.L., Ferreiro L., Ferreira R.R., Corbellini L.G., Deville M., Berthelemy M. \& Guillot J. 2011. Malassezia dermatitis in dogs in Brazil: diagnosis, evaluation of clinical signs and molecular identification. Veterinary Dermatology. 22(1): 46-52.

10 Namitome K., Kano R., Sekiguchi M., Iwasaki T., Kaneshima T. \& Nishifuji K. 2011. Isolation of Fusarium sp. from a Claw of a Dog with Onychomycosis. Journal of Veterinary Medical Science. 73: 965-969.

11 Nett C.S., Reichler I., Grest P., Hauser B. \& Reusch C.E. 2001. Epidermal dysplasia and Malassezia pachydermatis infection in West Highland White terriers. Veterinary Dermatology. 12: 285-90.

12 Scott D.W., Miller W.H. \& Griffin C.E. 2001. Fungal skin diseases. In: Scott D.W., Miller W.H. \& Griffin C.E. (Eds). Muller and Kirk's Small Animal Dermatology. 6th edn. Philadelphia: Saunders, pp.363-422.

13 Silva V., Moreno G.A., Zaror L., de-Oliveira E. \& Fischman O. 1997. Isolation of Malassezia furfur from patients with onicomicosis. Journal of Medical and Veterinary Mycology. 35: 73-74.

14 Velegraki A., Cafarchia C., Gaitanis G., Iatta R. \& Boekhout T. 2015. Malassezia infections in humans and animals: pathophysiology, detection, and treatment. Plos Pathogens. 11: e1004523. 